\title{
Signal-noise Ratio Recognition Algorithm Based on Singular Value Decomposition
}

\author{
Qiao $\mathrm{Yi}^{1, \mathrm{a}}$, Cui Qian ${ }^{2, \mathrm{~b}}$, Zhang Wei ${ }^{1, \mathrm{c}}$ and Liu Yan ${ }^{1, \mathrm{~d}}$ \\ ${ }^{1}$ Beijing Aerospace Control Center, Beijing 102206, China; \\ ${ }^{2}$ Xi'An Space Star Technology Enterprise (Group) Co. 710061, China; \\ aqiaoyi@bacc.org.cn, bcuiqian198611@163.com, 'czwbjhtc@sina.com, dvivianliuyan@gmail.com
}

Keywords: wireless communication, signal-noise ratio estimation, autocorrelation matrix, singular value decomposition.

\begin{abstract}
Signal-noise ratio is an important parameter in modern wireless communication systems and an important indicator to reflect signal quality. An accuracy signal-noise ratio can provide signal quality information required for power control and channel decoder, etc. This paper proposes a blind signal-noise ratio estimation algorithm, which makes use of singular value decomposition of signal autocorrelation matrix to estimate the signal-noise ratio. The algorithm does not require prior information related to the signal and has no special equipments on signal sampling rate and signal type. It is relatively easier, with a certain acceptable complexity to some estimated accuracy extent.
\end{abstract}

\section{Introduction}

Signal-noise ratio estimation is always important in the wireless communication field. On one hand, by estimating signal-noise ratio, an effective and adaptable demodulation algorithm can be adopted to improve demodulation capacity; on the other hand, in mobile communication, the system employs signal-noise ratio as measurement criteria for communication quality to provide channel quality information required for power control, etc. Commonly-used signal-noise ratio algorithms include data-aided and non-data-aided signal-noise ratio algorithms. However, as there is no information in the actual satellite communication to support the estimation of signal-noise ratio, the estimation of signal-noise ratio generally relies on non-data-aided signal-noise ratio algorithm.

In 1967, Benedict and Soong[1] early applied second-order moment and fourth-order moment to estimate the intensity of separation of carrier and noise in white Gaussian noise channel. In 1994, by using results of Benedict and Soong, Matzner [2] proposed in details a signal-noise ratio estimation method for multiplex channels. Pauluzzi [3] compared the signal-noise estimation algorithms of white Gaussian noise channel in his paper, among which the maximum-likelihood based estimation algorithm used decision feedback sequence or training sequence to construct the likelihood function. Although such algorithm could achieve a result with certain degree of precision when estimating the medium and high signal-noise ratio, it required more research steps and was highly complex for long data. The matrix-based method estimated used the relationship between second-order moment and fourth-order moment to estimate the signal- noise ratio. It performed well for signal sampled at a chip rate, but performed relatively badly for oversampling signals. Most literature was about statistic-based signal-noise ratio estimation algorithms, and few studied signal-noise ratio estimation algorithms based on joint frequency-phase modulation.

Based on those above, we applied another non-data-aided estimation algorithm: signal-noise ratio estimation algorithm based on singular value decomposition. This algorithm mainly uses the subspace [4] concept to distinguish the noise and the signal by decomposing the singular values, further estimating the signal-noise ratio. It has no special equipment on signal sampling rate and signal type, with a certain acceptable complexity to some estimated accuracy extent. 


\section{Signal model}

After sampling the lossless receiving signal through additive white Gaussian noise channel, the modulating signal $\mathrm{s}(\mathrm{t})$ can be expressed with the formula:

$$
y(n)=s(n)+w(n)
$$

Where, $y(n)$ is the receiving signal of Gaussian noise channel, $s(n)$ is the modulating signal with the type unknown, $w(n)$ is the white Gaussian noise with 0 of the mean value and $\sigma_{w}^{2}$ of the variance. The signal and the noise are independently distributed.

Autocorrelation matrix[10] of receiving signal is shown as follows:

$$
\begin{aligned}
R_{r r} & =E\left\{y(n) y(n)^{H}\right\}=E\left\{[s(n)+w(n)][s(n)+w(n)]^{H}\right\} \\
& =E\left\{s(n) s(n)^{H}\right\}+E\left\{w(n) w(n)^{H}\right\}=R_{s s}+R_{w w}
\end{aligned}
$$

Where, the order of the autocorrelation matrix is $\mathrm{m}$, and $\mathrm{H}$ is the conjugate transpose of the matrix. It can be seen from the above formula that the autocorrelation matrix of receiving signal $y(n)$ is equal to the sum of autocorrelation matrixes of the signal $s(n)$ and the noise $w(n)$, because the noise is separated from the signal and their cross-correlative function is 0 .

\section{Singular Value Decomposition(SVD) [5]}

After sampling the lossless receiving signal through additive white Gaussian noise channel, the modulating signal $\mathrm{s}(\mathrm{t})$ can be expressed with the formula:

As $R_{y y}, R_{s s}$ and $R_{w w}$ are symmetric, the following SVD can be conducted:

$$
R_{s s}=V \Lambda_{s} V^{H}
$$

Where, $\mathrm{V}$ is the orthogonal matrix. $\Lambda_{s}=\operatorname{diag}\left(\gamma_{1}, \gamma_{2}, \cdots, \gamma_{p}, 0, \cdots, 0\right)_{m \times m}$, among which $\operatorname{diag}(\gamma 1$, $\gamma 1, \gamma 2, \ldots, \gamma p, 0, \ldots, 0)$ represents the matrix $\gamma_{1} \geq \gamma_{2} \geq \cdots \geq \gamma_{p}$ which takes $(\gamma 1, \gamma 1, \gamma 2, \ldots, \gamma$ $\mathrm{p}, 0, \ldots, 0)$ as diagonal elements. $\mathrm{P}$ is less than the order of the matrix $\mathrm{m}$.

$$
R_{w w}=V \Lambda_{w} V^{H}
$$

Where,

$$
\begin{aligned}
& \Lambda_{w}=\operatorname{diag}\left(\sigma_{w}^{2}, \sigma_{w}^{2}, \cdots, \sigma_{w}^{2}\right)_{m \times m} \\
& R_{y y}=R_{s s}+R_{w w}=V\left(\Lambda_{s}+\Lambda_{w}\right) V^{H}=V \Lambda_{y} V^{H} \\
& \Lambda_{y}=\operatorname{diag}\left(\lambda_{1}, \lambda_{2}, \cdots, \lambda_{m}\right)_{m \times m} \\
& =\left(\begin{array}{cccccc}
\gamma_{1}+\sigma_{w}^{2} & 0 & & \cdots & & 0 \\
0 & \ddots & & & & \\
& \ddots & \gamma_{1}+\sigma_{w}^{2} & & & \vdots \\
\vdots & & & \sigma_{w}^{2} & \ddots & \\
& & & & \ddots & 0 \\
0 & & \ldots & & 0 & \sigma_{w}^{2}
\end{array}\right)_{m \times m}
\end{aligned}
$$

The space formed by the above $\mathrm{m}$ singular values is called as the space of signals and associated noises, among which the space formed by the first $\mathrm{p}$ singular values is called as the signal subspace and the space formed by the last m-p singular values is called as the noise subspace. It can prove that: the power $P_{s}$ of the signal s(n) is the sum of the each singular value, and the power ${ }^{w}$ of the noise $w(n)$ is m-tupling $\sigma_{w}^{2}$. Thus, the signal-noise ratio can be obtained with the following formula: 


$$
S N R=10 \log \left(p_{s} / p_{w}\right)=10 \log \frac{\sum_{k=1}^{p}\left(\lambda_{k}-\sigma_{w}^{2}\right)}{m \times \sigma_{w}^{2}}
$$

\section{Algorithm Implementation}

The receiving signal $\mathrm{y}(\mathrm{n})$ is used to estimate the correlation function sequence $\left\{\hat{r}_{0}, \hat{r}_{1}, \cdots, \hat{r}_{m-1}\right\}$ with the formula as follows:

$$
\hat{r}_{k}=E[y(n) y(n+k)]=\frac{1}{N} \sum_{i=0}^{N-1} y(i) y(i+k), k=0,1,2, \cdots, m-1
$$

Where, $\mathrm{N}$ is the length of the receiving sequence, $\mathrm{m}$ is the length of autocorrelation sequence of the signal. Choice of $\mathrm{m}$ value influences the autocorrelation matrix of receiving signal $y(n)$ and the resolution ratio of singular value expression signal, so it's very important to choose an appropriate $m$ value. Too small $\mathrm{m}$ results in high distortion of autocorrelation matrix, finally influencing estimation precision of signal-noise ratio. Too large $\mathrm{m}$ increases the unnecessary burden of calculation. According to our simulation experiment, m value can be generally selected from 50 to 100.

By using $\left\{\hat{r}_{0}, \hat{r}_{1}, \cdots, \hat{r}_{m-1}\right\}$, the autocorrelation matrix $\hat{R}_{y y}$ goes as follows:

$$
\hat{R}_{y y}=\left[\begin{array}{cccc}
\hat{r}_{0} & \hat{r}_{1} & \cdots & \hat{r}_{m-1} \\
\hat{r}_{1}^{*} & \hat{r}_{0} & \cdots & \hat{r}_{m-2} \\
\vdots & \vdots & \ddots & \vdots \\
\hat{r}_{m-1}^{*} & \hat{r}_{m-2}^{*} & \cdots & \hat{r}_{0}
\end{array}\right]
$$

Where, * represents the conjugate. As y(n) is real value sequence, $\hat{r}_{k}=\hat{r}_{k}^{*}$ the above formula can be simplified as:

$$
\hat{R}_{y y}=\left[\begin{array}{cccc}
\hat{r}_{0} & \hat{r}_{1} & \cdots & \hat{r}_{m-1} \\
\hat{r}_{1} & \hat{r}_{0} & \cdots & \hat{r}_{m-2} \\
\vdots & \vdots & \ddots & \vdots \\
\hat{r}_{m-1} & \hat{r}_{m-2} & \cdots & \hat{r}_{0}
\end{array}\right]
$$

By calculating singular value decomposition of $\hat{R}_{y y}$, the singular value sequence $\left\{\hat{\lambda}_{1} \geq \hat{\lambda}_{2} \geq \cdots \geq \hat{\lambda}_{m}\right\}$ is obtained. The dimension $\hat{p}$ of signal subspace and the dimension $\hat{m}_{\text {of }}$ space of signals and associated noises is determined. Estimation precision of dimension $\hat{p}$ of the signal subspace will directly influence the estimation effect of the algorithm.

In the practical application, band-limiting filtering and oversampling are processed before digitalization of the receiving signal. As the white Gaussian noise channel is generally of narrowband type, which is slightly wider than the signal bandwidth, the dimension $\hat{m}<m$ (where $m$ is the number of steps of the autocorrelation matrix) of the space of signal and the associated noise. The average power of each harmonic wave of the noise can be calculated by the following formula:

$$
\hat{\sigma}_{w}^{2}=\frac{1}{\hat{m}-\hat{p}} \sum_{i=\hat{p}+1}^{\hat{m}} \hat{\lambda}_{i}
$$

It can be seen that $\hat{p}$ and $=\hat{m}$ can directly influence the calculation results of the signal-noise ratio, thus determination of $\hat{p}$ and $\hat{m}$ is critical to estimation of the signal-noise ratio. By differencing singular value sequence, the gradient sequence can be obtained. It can be observed that there are obvious break points in the corresponding locations of $\hat{p}$ and $\hat{m}$. Thus, $\hat{p}$ and $\hat{m}$ can be determined by estimating locations of the break points. 
The signal-noise ratio of the modulated signal can be obtained by the formula:

$$
S \hat{N} R=10 \log \frac{\sum_{i=1}^{\hat{p}}\left(\hat{\lambda}_{i}-\hat{p} \times \hat{\sigma}_{w}^{2}\right)}{\hat{m} \times \hat{\sigma}_{w}^{2}}
$$

\section{Simulation Performance}

Take the white Gaussian noise channel as an example, modulation type of the signal is selected as QPSK and the simulation software uses Matlab Ver7.11. This paper mainly starts from estimation range of the signal-noise ratio to study the influence on estimation performance of the algorithm.

Actual signal-noise ratio of the signal increases from initially $5 \mathrm{~dB}$ to $30 \mathrm{~dB}$. Fig. 1 shows the comparison chart of the actual signal-noise ratio and the estimated signal-noise ratio, and Fig. 2 is the varying curvature of normalized mean squared error between actual signal-noise ratio and the estimated signal-noise ratio vs. signal-noise ratio. The two figures show that along with an increase in actual value of the signal-noise ratio, the error between the actual and the estimated signal-noise ratio is gradually increased. Thus, make sure that the signal-noise ratio falls within $25 \mathrm{~dB}$.
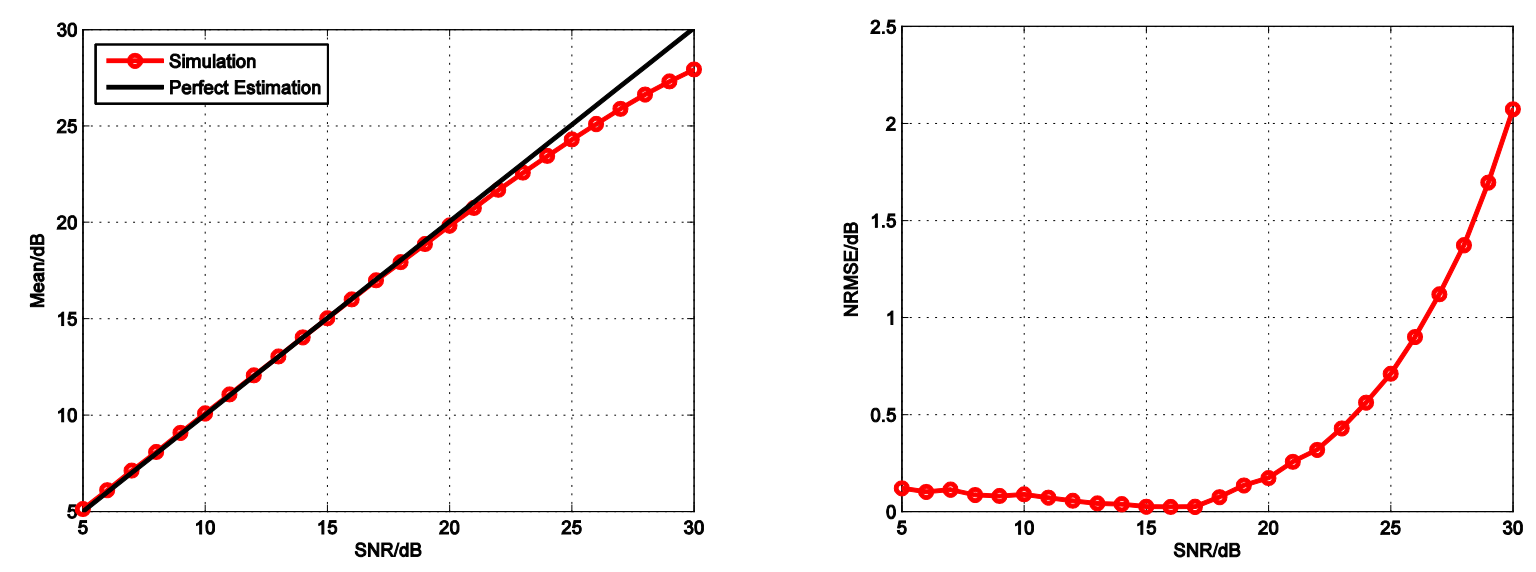

Fig. 1 sample curve of estimated value of signal-noise ratio and varying curvature of estimated NMSE signal-noise ratio

\section{Conclusion}

This paper proposes an estimation algorithm for signal-noise ratio based on singular value decomposition, which is not subject to prior information of the signal and works more precisely to a certain signal-noise ratio extent for signals with different modulation type. However, a further study is needed about how to acquire good autocorrelation matrix length $\mathrm{m}$ and how to obtain good recognition results under the circumstance of large signal-noise ratio.

\section{References}

[1] Benedict, T. R., \& Soong, T. T. (1967). The joint estimation of signal and noise from the sum envelope. Information Theory, IEEE Transactions on, 13(3), 447-454.

[2] Matzner, R., \& Englberger, F. (1994, August). An SNR estimation algorithm using fourth-order moments. In Information Theory, 1994. Proceedings., 1994 IEEE International Symposium on (p. 119).

[3] Pauluzzi, D. R., \& Beaulieu, N. C. (2000). A comparison of SNR estimation techniques for the AWGN channel. Communications, IEEE Transactions on, 48(10), 1681-1691. 
[4] Moulines, E., Duhamel, P., Cardoso, J. F., \& Mayrargue, S. (1995). Subspace methods for the blind identification of multichannel FIR filters. Signal Processing, IEEE Transactions on, 43(2), 516-525.

[5] Malioutov, D., Çetin, M., \& Willsky, A. S. (2005). A sparse signal reconstruction perspective for source localization with sensor arrays. Signal Processing, IEEE Transactions on, 53(8), 3010-3022. 